\title{
ON THE DYNAMICS OF A SECOND-ORDER THIN ROD*
}

\author{
$\mathrm{BY}$ \\ FRANCO PASTRONE \\ Università di Torino
}

\begin{abstract}
A second-order theory of hyperelastic thin rods is developed from a threedimensional point of view. The basic topics of geometry and kinematics are given and strain variables are introduced. By means of the virtual work theorem we obtain the equations of motion; the internal work theorem determines the choice of stress variables and, under the hypothesis of hyperelasticity, stress-strain relations are shown. Finally a suitable choice of a second-degree potential, in the Signorini sense, is given.
\end{abstract}

1. Introduction. Following up a previous paper of Antman and Warner [1], where a three-dimensional theory of rods was developed, Benenti $[2,3]$, and the author $[5,6]$, studied the problem of a first-order theory of hyperelastic thin rods with perfect internal constraints. There was, however, a deficiency in this theory, since for the simple problem of pure flexure the exact displacements cannot be included in that theory, as Green, Laws, and Naghdi showed in [4].

To avoid this obstacle we introduce a second-order theory, according to Antman and Warner's theory, which allows us to introduce a second-degree strain-energy function by means of Signorini's theory of second-degree elasticity (see [7, 8]).

2. Geometry. Let $C$ be a three-dimensional continuum body and $l$ a material curve in it with parametrical equations $O Q=O Q\left(x^{3}, t\right),\left(x^{\alpha}, x^{3}\right)$ material coordinates, $\mathbf{g}_{i}=\partial_{i} O P$ the associated basis. (Latin indices range over 1, 2, 3; Greek indices over 1, 2; the summation convention holds in each case; $\partial_{i} \equiv \partial / \partial x^{i}$ ). We define $C$ as a second-order rod, according to $[1,2,3]$, if it can be described by the equation:

$$
O P=O Q\left(x^{3}, t\right)+x^{\alpha} \mathbf{a}_{\alpha}\left(x^{3}, t\right)+\frac{1}{2} x^{\alpha} x^{\beta} c_{\alpha \beta}{ }^{i} \mathbf{a}_{i}\left(x^{3}, t\right),
$$

where $O$ is a fixed point, $\mathbf{a}_{i}=\left(\mathbf{g}_{i}\right)_{x} \alpha_{=0}$, and $c_{\alpha \beta}{ }^{i}$ are the connection coefficients on the curve $l$ defined by $c_{\alpha \beta}{ }^{i} \mathbf{a}_{i}=\left(\partial^{2} O P / \partial x^{\alpha} \partial x^{\beta}\right)_{x} \alpha_{=0}$. In fact the coefficients $c_{\alpha \beta}{ }^{i}$ are related to the connection coefficients $\Gamma_{i j}{ }^{h}=\partial_{i} \mathbf{g}_{j} \cdot \mathbf{g}^{h}$ through $c_{\alpha \beta}{ }^{i} \mathbf{a}_{i}=\left(\Gamma_{a b}{ }^{i}\right)_{x} \alpha_{=0} \mathbf{a}_{i}$. Moreover, the coefficients $k_{i}{ }^{j}=\partial_{3} \mathbf{a}_{i} \cdot \mathbf{a}^{j}$ that appear in the linear theory (see [2]) can be included in ours by means of the general definition:

$$
c_{i j}{ }^{h} \mathbf{a}_{h}=\left(\partial^{2} O P / \partial x^{i} \partial x^{j}\right)_{x} \alpha_{=0} \rightleftarrows c_{i j}{ }^{h}=\left(\partial_{i} \boldsymbol{g}_{j}\right)_{x} \alpha_{=0} \cdot \mathbf{a}^{h},
$$

from which it follows that $k_{i}{ }^{j}=c_{3 i}{ }^{j}$.

\footnotetext{
* Received February 7, 1977. The research reported in this paper was supported by the GNFM of the Italian C.N.R.
} 
It is easy to show that the following relations hold:

$$
\begin{aligned}
\mathbf{g}_{i} & =\mathcal{E}_{i}{ }^{j} \mathbf{a}_{j} \\
\mathcal{E}_{\alpha}{ }^{i}=\delta_{\alpha}{ }^{i}+x^{\beta} c_{\beta \alpha}{ }^{i}, \quad \mathcal{E}_{3}{ }^{i} & =\delta_{3}{ }^{i}+x^{\beta} c_{3 \beta}{ }^{i}+\frac{1}{2} x^{\alpha} x^{\beta} \nabla_{3} c_{\alpha \beta},
\end{aligned}
$$

$\nabla_{3}$ being the "covariant derivative" on $l$ defined by

$$
\nabla_{3} c_{h l}{ }^{i}=\partial_{3} c_{h l}{ }^{i}+c_{3 j}{ }^{i} c_{h l}{ }^{j}
$$

and $\delta_{j}{ }^{i}$ the Kronecker tensor.

We can now give explicit expressions for volume and surface elements $d C$ and $d \Sigma$ of our body. Note that in the following $Q\left(x^{3}\right)$ will be the region of the Cartesian $\left(x^{1}, x^{2}\right)$-plane over which the curvilinear coordinates $x^{\alpha}$ range as surface parameters for the cross-section $\Sigma$ determined by $x^{3}=$ const. The element of volume $d C$ is given by

$$
d C=\sqrt{ } g d x^{1} d x^{2} d x^{3},
$$

where

$$
g=\operatorname{det}\left\|g_{i j}\right\|=\mathbf{g}_{1} \times \mathbf{g}_{2} \cdot \mathbf{g}_{3},
$$

or, by using (2):

$$
\sqrt{ } g=\eta_{i j k} \varepsilon_{1}{ }^{i} \varepsilon_{2}{ }^{j} \varepsilon_{3}{ }^{k} \mathbf{a}_{1} \times \mathbf{a}_{2} \cdot \mathbf{a}_{3}
$$

$\eta_{i j k}$ being the Ricci tensor; according to the impenetrability principle it is

$$
\eta_{i j k}{E_{1}}^{i}{\mathcal{E}_{2}}^{j}{\mathcal{E}_{3}}^{k}>0 \text {. }
$$

Let us introduce the normal unit vector to the cross-section $\Sigma: \mathrm{n}(P)=\tilde{g}^{-1 / 2} \mathbf{g}_{1} \times \mathbf{g}_{2}(\tilde{g}$ $\left.=\operatorname{det}\left\|g_{\alpha \beta}\right\|\right)$ and the tangent to the curve $l: \mathrm{t}=(1 / \epsilon) \mathrm{a}_{3}$, with $\epsilon=d s / d x^{3}=\left(a_{33}\right)^{1 / 2}>0(s$ arc length of 1). Then, introducing

$$
\gamma=\mathfrak{t} \cdot \mathrm{n}, \quad \mathcal{E}=\gamma \eta_{i j k} \varepsilon_{1}{ }^{i} E_{2}{ }^{j} E_{3}{ }^{k}, \quad D=(1 / \epsilon)(g / \tilde{g})^{1 / 2},
$$

we obtain

$$
d C=\epsilon \sqrt{ } \tilde{a} \& d x^{1} d x^{2} d x^{3}, \quad d \Sigma=\sqrt{g} d x^{1} d x^{2}, \quad\left(\sqrt{a}=\operatorname{det}\left\|a_{\alpha \beta}\right\|\right),
$$

and finally

$$
d C=\epsilon D d \Sigma d x^{3} .
$$

Formally we obtain the same expression as in the linear case (nine parameter rod, see $[2,3])$ and if we reduce our analysis to such a case, i.e. if we suppose $c_{\rho \sigma}{ }^{i}=0$, we find the formula (20) in [2]: $\mathfrak{D}=(D)_{c_{\rho \sigma}} i_{=0}=\gamma\left(1+x^{\alpha} k_{\alpha}{ }^{3}\right)$, being $c_{3 \alpha}{ }^{3}=k_{\alpha}{ }^{3}$.

3. Equations of motion. In order to reach the equations of motion, we shall use the relation valid, as shown in [2], for dynamics of classical three-dimensional bodies:

$$
\int_{C} k F \cdot \xi d C+\int_{\partial C} \phi \cdot \xi d \partial C+\int_{c} \boldsymbol{\phi}^{i} \cdot \partial_{i} \xi d C=0,
$$

where $k$ is the density, $\mathbf{F}=\mathbf{g}-\mathbf{a}$, the difference between the body force per unit mass $\mathbf{g}$ and the acceleration a of the point $P, \phi$ the surface force on the external boundary, $\boldsymbol{\phi}^{i}$ the internal stresses; (8) must hold for every arbitrary choice of the vector function $\xi$. Let us take: 


$$
\xi=\xi\left(x^{3}\right)+x^{\alpha} \xi_{\alpha}\left(x^{3}\right)+\frac{1}{2} x^{\alpha} x^{\beta} \xi_{\alpha \beta}\left(x^{3}\right),
$$

$\xi, \xi_{\alpha} \xi_{\alpha \beta}$ being arbitrary vector functions of $x^{3}$, and introduce for the acceleration $a$ the expression

$$
\mathbf{a}=\dot{\mathbf{w}}+x^{\alpha} \dot{\mathbf{w}}_{\alpha}+\frac{1}{2} x^{\alpha} x^{\beta} \dot{\mathbf{w}}_{\alpha \beta},
$$

with

$$
\mathbf{w}=O Q, \mathbf{w}_{\alpha}=\dot{\mathbf{a}}_{\alpha}, \mathbf{w}_{\alpha \beta}=\frac{d}{d t}\left(c_{\alpha \beta}{ }^{i} \mathbf{a}_{i}\right) .
$$

Then, by a suitable integration by parts over the cross-sectional area $\Sigma$, we can obtain the following equations of motion (see, as an example, [2]):

$$
\begin{aligned}
& \rho\left(\mathbf{f}-\dot{\mathbf{w}}+y^{\rho} \dot{\mathbf{w}}_{\rho}-\frac{1}{2} y^{\rho \varphi} \dot{\mathbf{w}}_{\rho \varphi}\right)-1 / \epsilon \partial_{3}\left(\epsilon \mathbf{R}^{3}\right)=0, \\
& \rho\left(\mathbf{m}^{\alpha}-y^{\alpha \alpha} \dot{\mathbf{w}}-y^{\alpha \mu} \dot{\mathbf{w}}_{\rho}-\frac{1}{2} y^{\alpha \rho \phi} \dot{\mathbf{w}}_{\rho \varphi}\right)+\mathbf{R}^{\alpha}-1 / \epsilon \partial_{3}\left(\epsilon \mathbf{M}^{\alpha 3}\right)=0, \\
& \left.\rho\left(\mathbf{m}^{\alpha \beta}-\frac{1}{2} !^{\alpha \beta \beta} \dot{\mathbf{w}}-\frac{1}{2} y^{\alpha \rho \phi} \dot{\mathbf{w}}-\frac{1}{4} y^{\alpha \beta \rho \phi} \mathbf{w}_{\rho \varphi}\right)+\mathbf{M}^{\alpha \beta}-1 / \epsilon \partial_{3}(\epsilon)^{\alpha \beta}\right)=0,
\end{aligned}
$$

with the boundary conditions:

$$
\left.\varphi_{0 . L} \pm\left[\epsilon \mathbf{R}^{3}\right]_{0}{ }^{l .}=0 ; \varphi_{0, L^{\alpha}} \pm\left[\epsilon \mathbf{M}^{\alpha 3}\right]_{0}{ }^{L}=0 ; \quad \varphi_{0, L}{ }^{\alpha \beta} \pm[\epsilon \mathfrak{M})^{\alpha \beta}\right]_{0}{ }^{L}=0 .
$$

In formulas (10) and (11) we introduced:

$$
\begin{aligned}
& \rho=\int_{Q} k D d \Sigma \quad \text { linear density, } \\
& y^{\alpha_{1} \alpha_{2} \cdots \alpha_{m}}=\frac{1}{\rho} \int_{Q \mathcal{R}} x^{\alpha_{1}} x^{\alpha_{2}} \cdots x^{\alpha_{m} D} D \Sigma \quad n \text { th-order inertia tensor, } \\
& \mathrm{f}=\frac{1}{\rho} \int_{Q \mathcal{Q}} k \mathbf{g} D d \Sigma \quad \text { body force resultant vector, } \\
& \mathrm{m}^{\alpha_{1} \ldots \alpha_{m}}=\frac{1}{n !} \int_{Q} k \mathbf{g} x^{\alpha_{1}} \cdots x^{\alpha_{m}} D d \Sigma \quad n \text { th-order body force momentum, } \\
& \mathbf{R}^{i}=\int_{Q} \boldsymbol{\Phi}^{i} D d \Sigma \quad \text { stress-resultant vector, } \\
& \mathbf{M}^{\alpha i}=\int_{Q} x^{\alpha} \mathbf{\Phi}^{i} D d \Sigma \quad \text { first-order internal stress momentum, } \\
& : \pi \pi^{\alpha \beta}=\frac{1}{2} \int_{Q} x^{\alpha} x^{\beta} \mathbf{\Phi}^{3} D d \Sigma \quad \text { second-order internal stress momentum, } \\
& \boldsymbol{\phi}_{o, l}=\left[\int_{Q} \phi D d \Sigma\right]_{o, l} ; \quad \boldsymbol{\phi}_{o, l}{ }^{\alpha_{1}} \cdots \alpha_{m}=\left[1 / n ! \int_{Q} x^{\alpha_{1}} \cdots x^{\alpha_{m}} \phi D d \Sigma\right]_{O, L} .
\end{aligned}
$$

Eqs. (10) are 18 partial differential equations in the 18 kinematical unknowns $a_{i}, c_{\alpha \beta}{ }^{i}=$ $c_{\beta \alpha}{ }^{i}$, and we can get a complete scheme as soon as we add constitutive equations by introducing a suitable strain-energy function compatible with this second-order theory, as we shall show in the following section.

Before doing this we want to point out that reactions do not appear in the equations of motion, as it is natural in our scheme; however, for a detailed discussion about the internal 
constraints and relative reactions, we refer to [1, p. 153]; at any rate, this is an approximate scheme obtained by truncation of suitable expansions, without introducing internal constraints.

The kinematical variables $\mathbf{a}_{i}, c_{\alpha \beta}{ }^{i}$ may be replaced by the variables $a_{i j}=\mathbf{a}_{i} \cdot \mathbf{a}_{j}$ and $c_{i \alpha}{ }^{j}$; in fact, the differential system

$$
\partial_{3} \mathbf{a}_{i}=c_{3 i}{ }^{j} \mathbf{a}_{j}
$$

admits one and only one solution if we know the variables $c_{3 i}{ }^{j}$. But it is:

$$
c_{33}{ }^{i}=\frac{1}{2}\left(a^{3 i} \partial_{3} a_{33}+a^{i \alpha} \partial_{3} a_{3 \alpha}-a^{i \alpha} c_{3 \alpha}{ }^{j} a_{j 3}\right),
$$

so, if we assign the variables $a_{i j}$ and $c_{3 \alpha}{ }^{j}$ satisfying the compatibility conditions

$$
\partial_{3} a_{\alpha \beta}=c_{3 \alpha}{ }^{h} a_{\beta h}+c_{3 \beta}{ }^{k} a_{\alpha k},
$$

we can determine univocally the vectors $\mathbf{a}_{i}$. Afterwards it will be useful to introduce also the derivatives $\nabla_{3} c_{\rho_{\sigma}}{ }^{i}$ as variables, but obviously if we know the $a_{i j} \mathrm{~s}$ and $c_{i \alpha}{ }^{j} \mathrm{~s}$ we know the $\nabla_{3} c_{\rho_{\sigma}}{ }^{\prime}$ s as well. Finally we note that the relations (13) are the only compatibility conditions available, as the compatibility tensor $\mathbf{R}_{i j h}=\partial_{i} \partial_{j} \mathbf{g}_{h}-\partial_{j} \partial_{i} \mathbf{g}_{h}$ vanishes identically making use of the relations (2), (3), in which conditions (13) appear implicitly through (12).

4. Constitutive relations. In the equations of motion (10) the stress is given by the vectors

$$
\begin{gathered}
\mathbf{R}^{i}=R^{i j} \mathbf{a}_{j}=R_{j}{ }^{i} \mathbf{a}^{j}, \\
\mathbf{M}^{\alpha i}=M^{\alpha i j} \mathbf{a}_{j}=M^{\alpha i} \mathbf{a}^{j}, \\
\mathfrak{T}^{\alpha \beta}=\mathfrak{M}^{\alpha \beta j} \mathbf{a}_{j}=\mathscr{T}^{\alpha \beta}{ }_{j} \mathbf{a}^{j},
\end{gathered}
$$

the components of which are not all independent, since the symmetry relation holds: $\phi^{i} \times$ $\mathbf{g}_{i}=0$, and by integration over the cross-section $\Sigma$, the symmetry of the following object follows:

$$
S^{i k}=R^{i k}+M^{\rho j k} c_{\rho j}{ }^{i}+\mathfrak{T}^{\rho \sigma k} \nabla_{3} c_{\rho_{\sigma}}{ }^{i}=S^{k i} .
$$

Moreover the symmetry relations hold: $\mathbf{M}^{\alpha \beta}=\mathbf{M}^{\beta \alpha}, M^{\alpha i j}=M^{\alpha j i}$, $\mathfrak{T}^{\alpha \beta}=\mathfrak{M}^{\beta \alpha \kappa}$, so that the components given by (14) reduce to 24 independent components. The expression of the virtual work of internal stresses

$$
\partial \mathscr{L}^{\prime \prime}=\int_{c} \boldsymbol{\phi}^{i} \cdot \mathbf{g}_{i} d C=\int_{0}^{L}\left[\frac{1}{2} S^{i k} \partial a_{i k}+M_{k}{ }^{\alpha j} \partial c_{\alpha j}{ }^{k}+\mathfrak{M}_{k}{ }^{\alpha \beta} \partial\left(\nabla_{3} c_{\alpha \beta^{k}}\right)\right] \epsilon d x^{3},
$$

obtained, as usual, by integration over the cross-section $\Sigma$, determines the choice of the tensor function $S^{i k}, M^{\alpha j}, \mathfrak{T}^{\alpha \beta}{ }_{k}$ as stress variables and the differences:

$$
E_{i k}=\frac{1}{2}\left(a_{i k}-A_{i k}\right), \quad F_{\alpha i}{ }^{k}=\frac{1}{2}\left(c_{\alpha i}{ }^{k}-C_{\alpha i}{ }^{k}\right), \quad L_{\alpha \beta}{ }^{i}=\frac{1}{2}\left(\nabla_{3} c_{\alpha \beta}{ }^{i}-\nabla_{3}{ }^{*} C_{\alpha \beta}{ }^{i}\right),
$$

where $A_{i k}, C_{\alpha \beta}{ }^{i}, \nabla_{3}{ }^{*}$ are $a_{i k}, c_{\alpha \beta}{ }^{i}, \nabla_{3}$ in the reference configuration, as strain variables.

If we make the hypothesis of hyperelasticity for the body, i.e. we admit there exists a three-dimensional elastic isothermal potential $\mathscr{F}=\mathscr{F}(E / F / L)$, referred to the unit volume, the following equality must hold: $\boldsymbol{\phi}^{i} \cdot \partial \mathbf{g}_{i}=-k \partial \mathscr{F}$. By integration between two arbitrary cross-sections $\Sigma_{i}, \Sigma_{2}$ we obtain:

$\int_{x_{1}{ }^{3}{ }^{3}}^{x_{1,2}{ }^{3}}\left[\frac{1}{2} S^{i k} \partial a_{i k}+M^{\alpha i}{ }_{k} \partial c_{\alpha i}{ }^{k}+\mathscr{T}^{\alpha \beta_{k}} \partial\left(\nabla_{3} c_{\alpha \beta}{ }^{i}\right)\right] \epsilon d x^{3}=-\partial \int_{x_{1,}{ }^{3}}^{x_{22}{ }^{3}}\left[\int_{\Sigma} k \mathcal{F} D d \Sigma\right] \epsilon d x^{3},(17)$ 
where the linear operator $\partial$ is permutable with the integral, as shown in [3]. It is natural to introduce now the one-dimensional elastic isothermal potential $\varphi=\varphi(E / F / L)$, referred to the unit length, by means of

$$
\varphi=1 / \rho \int_{\Sigma} k \mathfrak{F} D d \Sigma
$$

so that from (14) we have

$$
-\partial \int_{x_{(1)}{ }^{3}{ }^{3}}^{x_{12}{ }^{3}} \rho \varphi \epsilon d x^{3}=-\int_{x_{(1,1}{ }^{3}}^{x_{(2)}{ }^{3}} \rho \partial \varphi \epsilon d x^{3}
$$

and hence

$$
\frac{1}{2} S^{i k} \partial a_{i k}+M_{k}{ }^{\alpha i} \partial c_{\alpha i}{ }^{k}+\mathfrak{T}_{k}{ }^{\alpha \beta} \partial\left(\nabla_{3} c_{\alpha \beta}{ }^{k}\right)=-\rho \partial \varphi .
$$

By developing the "differential" $\partial \varphi$ and using relations (16), we immediately reach the constitutive equations:

$S^{i k}=-\rho\left(\partial \varphi / \partial E_{i k}\right), \quad M_{k}{ }^{\alpha i}=-\frac{1}{2} \rho\left(\partial \varphi / \partial F_{\alpha i}{ }^{k}\right), \quad \mathscr{T}_{k}{ }^{\alpha \beta}=-\frac{1}{2} \rho\left(\partial \varphi / \partial L_{\alpha \beta}{ }^{k}\right)$.

Therefore, it is enough to assign a suitable three-dimensional potential to get a complete scheme, as we shall do in the next section.

5. Second-degree potential. Since we are dealing with a second-order theory, we want to point out second-order effects; we have to choose a second-degree potential, or, better still in our case, a second-degree strain-energy function.

We shall now follow the approach of Signorini [7, 8] as indicated in Stazi [9], under the hypothesis that the actual three-dimensional body with a rod structure is an isotropic system subject to a reversible isothermal process. In this case it can be shown (see [9]) that the strain-energy function is a function of the strain variables through the invariants

$$
L=\epsilon_{i}{ }^{i}, \quad Q=\epsilon_{j}{ }^{i} \epsilon_{i}{ }^{j}, \quad C=\epsilon_{j}{ }^{i} \epsilon_{h}{ }^{j} \epsilon_{i}{ }^{h},
$$

that is

$$
\mathcal{F}=\mathscr{F}(L, Q, C) .
$$

If we impose the function $\mathcal{F}$ to make strain-stress relations exactly quadratic, then the strain-energy function must be given by the following formula (see [9]):

$$
\mathcal{F}=H\left[\nu\left(I_{1}-1\right)+\frac{\lambda-2 \mu+3 \nu}{2} I_{1}^{2}+2(\nu-\mu) I_{2}\right]+\nu,
$$

where we put $I_{1}, I_{2}$ strain-invariant, related to the invariants (19) by

$$
\begin{aligned}
& L=I_{1}, \quad Q=I_{1}{ }^{2}-2 I_{2}, \\
& C=I_{1}{ }^{3}-3 I_{1} I_{2}+\frac{3}{8}\left(H^{2}-1-2 I_{1}-4 I_{2}\right),
\end{aligned}
$$

and moreover:

$$
H=\left(1+2 L+2 L^{2}-2 Q+4 / 3 L^{3}-4 L Q+8 / 3 C\right)^{1 / 2} ;
$$

$\lambda, \mu$ are Lamé constants, $\nu$ a constant satisfying the conditions: $3 \lambda+2 \mu>5 \nu, \mu>\nu, \nu>0$. If $\nu=0$ we find the usual linearized potential, within the well-known conditions $3 \lambda+2 \mu>$ $0, \mu>0$. 
To compute explicitly the invariants (19) we recall that $\epsilon_{j}{ }^{l}=g^{i k} \epsilon_{j k}=\frac{1}{2} g^{i k}\left(g_{j k}-G_{j k}\right)$. If we suppose that in the reference configuration the curve $l_{0}$ is rectilinear and every crosssection $\Sigma_{0}$ is plane, then $G_{i j}=A_{i j}$, while the $g_{i j}$ s can be obtained from (2), (3) and the $g^{i j} \mathrm{~s}$ from the analogous ones:

$$
\mathbf{g}^{i}=\rho_{k}{ }^{i} \mathbf{a}^{k}
$$

where

$$
\mathcal{S}_{k}{ }^{i}=\gamma / \epsilon \epsilon_{j h l} \mathcal{E}_{k+1}{ }^{h} \mathcal{E}_{k+2}{ }^{l} \quad(k+r \bmod 3) .
$$

Substituting these expressions in (19) and thereafter in (20), we obtain the three-dimensional second-order strain-energy function in terms of actual strain variables $E_{i j}, F_{\alpha k}{ }^{i}$, $L_{\alpha \beta}{ }^{i}$, from which, by integration, we get the one-dimensional second-order potential and the consequent second-order constitutive equations.

\section{REFERENCES}

[1] S. S. Antman and W. H. Warner, Arch. Rat. Mech. Anal. 23, 135 (1966)

[2] S. Benenti, Rend. di Matem. 4 (IV), 705 (1971)

[3] S. Benenti, Rend. Sem. Matem. Univ. Polit. Torino 31, 243 (1973)

[4] A. E. Green, N. Laws, and P. M. Naghdi, Arch. Rat. Mech. Anal. 25, 185 (1967)

[5] F. Pastrone, Rend. Sem. Matem. Univ. Polit. Torino 31, 379 (1973)

[6] F. Pastrone, Rend. Sem. Matem. Univ. Polit. Torino 33, 181 (1975)

[7] A. Signorini, Annali Matem. Pura Appl. S. IV, 30 (1949)

[8] A. Signorini, Intern. Kong. Tekn. Mek., Stockholm (1930)

[9] L. Stazi, Rend. Sem. Matem. Univ. Polit. Torino 33, 313 (1975) 